\title{
Spontaneous Regression and Reoccurrence of Osteosarcoma in a Canine Tibia
}

\author{
Olivia Obringer ${ }^{1}$ Bradley Coolman ${ }^{2}$ Jason Crawford ${ }^{3}$
}

1 University of Saint Francis, Fort Wayne, Indiana, United States

${ }^{2}$ Northeast Indiana Veterinary Emergency \& Specialty Hospital, Fort

Wayne, Indiana, United States

${ }^{3}$ Inner-Pet Imaging, Huntertown, Indiana, United States
Address for correspondence Bradley Coolman, DVM, MS, DACVS, Northeast Indiana Veterinary Emergency \& Specialty Hospital, 5818 Maplecrest Road, Fort Wayne, IN, 46835, United States (e-mail: cooldvm@gmail.com).

VCOT Open 2021;4:e86-e91.

Abstract

Keywords
- dog
- spontaneous tumour
regression
- osteosarcoma
- tibial plateau levelling
osteotomy

A 32-month-old female Labrador Retriever mix-breed presented for right pelvic limb lameness with firm swelling on the lateral aspect of the stifle. Radiographs revealed a mass with periosteal elevation along the lateral cortex of the proximal tibia. Histology on a core biopsy sample was diagnostic for osteosarcoma. Surgical treatment was declined. Eight months post diagnosis, the patient was re-evaluated by the primary care veterinarian and had no lameness, pain, or tibial swelling. Sixty-three months later (5.2 years), the patient presented for cranial cruciate ligament ruptures. Radiographs revealed a smooth bony protuberance on the lateral aspect of the right proximal tibia in the area of the previous osteosarcoma. The patient underwent bilateral tibial plateau levelling osteotomies. The surgical recovery was uneventful with normal healing. Twenty-seven months following surgery, the patient returned for painful swelling around the right proximolateral tibia. Radiographs revealed an $8 \times 9 \mathrm{~cm}$ osteoproliferative and osteolytic lesion of the proximal tibia. Osteosarcoma was confirmed via core biopsy. The bone tumour grew rapidly, and the patient was euthanatized 3 months thereafter. This case report demonstrates the unusual occurrence of an osteosarcoma in a young dog which spontaneously regressed. Ninety months (7.5 years) after clinical resolution of the initial tumour, osteosarcoma formed again in the same location. Lifetime patient monitoring with repeated clinical exams, serial radiographs, and multiple biopsies by the same surgeon, pathologists, and radiologist are unique features of this case report.

\section{Introduction}

Osteosarcoma (OS) is the most common primary bone tumour in dogs, usually affecting large breeds on the average between 7 and 9 years and a median of 7 years. ${ }^{1-13}$ Tumours typically develop on the appendicular skeleton and metastasis can occur very quickly, even despite early amputation. $^{3-5,14,15}$ Surgical trauma, such as amputation, can increase the number of circulating cancer cells through shedding and tissue manipulation. ${ }^{16}$ Thoracic limb OS is

received

December 18, 2020

accepted after revision June 16, 2021
DOI https://doi.org/ $10.1055 / \mathrm{s}-0041-1735844$. ISSN 2625-2325. diagnosed twice as often as OS on pelvic limbs; clinical symptoms include lameness, local pain and swelling of the affected limb. ${ }^{5,13}$ Treatment options typically begin with surgical excision of the entire osseous compartment containing tumour, followed by chemotherapy. This may result in 1 to 2 years survival time. ${ }^{6}$ If thoracic radiographs reveal metastases, amputation and chemotherapy will do little to slow the disease process. ${ }^{15}$ Affected dogs have a median survival time of 1 to 3 months without treatment and with

\section{(C) 2021. The Author(s).}

This is an open access article published by Thieme under the terms of the Creative Commons Attribution License, permitting unrestricted use, distribution, and reproduction so long as the original work is properly cited. (https://creativecommons.org/licenses/by/4.0/) Georg Thieme Verlag KG, Rüdigerstraße 14, 70469 Stuttgart, Germany 
analgesics only. ${ }^{17}$ Spontaneous regression of appendicular OS is rarely observed and only four such cases are reported in dogs, ${ }^{1}$ and a very few in humans. ${ }^{18-22}$ Fracture-induced or metallic implant-associated OS in canine patients has been recorded more often throughout the years, yet there is little known on etiology. ${ }^{12,23-27}$ This present case report describes the occurrence of a tibial OS occurring in a 32-month-old dog, spontaneously resolving initially, and then reappearing in the same location 27 months after a tibial plateau levelling osteotomy (TPLO), that is 97 months ( 8 years) after the first appearance. The dog was euthanatized due to rapid tumour growth and declining health 3 months after the second bone tumour diagnosis at the age of 11 years.

\section{Case Description}

A 32-month-old spayed female Labrador Retriever mixbreed weighing $34 \mathrm{~kg}$ presented for right pelvic limb lameness and a $6 \mathrm{~cm}$ swelling lateral to the proximal tibia, which was firm and painful upon palpation. No known trauma was reported. Thoracic radiographs were normal. Radiographs of the right tibia revealed a mass originating from the metaphysis with periosteal elevation ( - Fig. 1). A needle aspirate cytology was performed and showed a monotonous population of mesenchymal cells with malignant criteria. Three core biopsy samples were obtained from the tumefaction on the lateral aspect of the right tibia via an 11-gauge Jamshidi instrument. The incision was closed with skin staples. Histologically, the bone lesion consisted of a dense proliferation of polygonal to spindle cells with indistinct cell limits, pale eosinophilic cytoplasm, oval to round nuclei, indistinct nucleoli and rare mitotic figures (-Fig. 2). The samples revealed abundant partially mineralized osteoid closely associated with neoplastic cells. Necrosis was extensive. Morphologic diagnosis was OS.

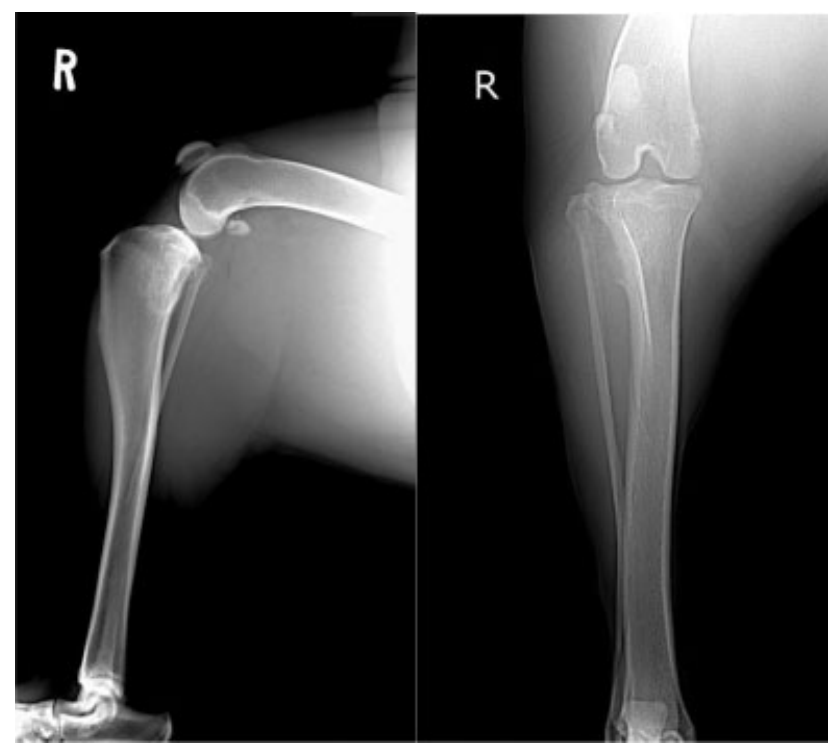

Fig. 1 Orthogonal right stifle radiographs of a 32-month-old canine female revealing a smooth periosteal elevation and bony reaction on the lateral tibial metaphysis.

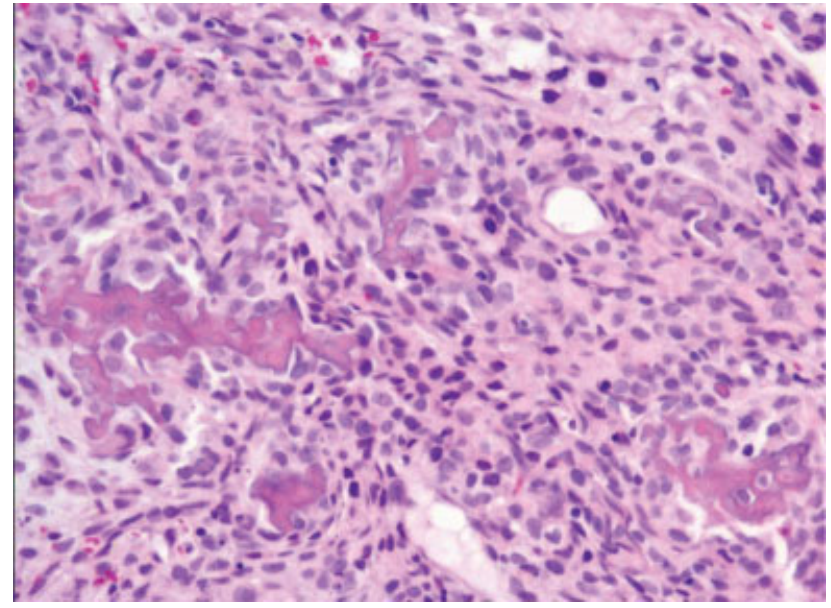

Fig. 2 Histopathology figure $(400 \times$ magnification) from the initial right tibial bone biopsy. The lesion consists of a dense proliferation of mostly spindle cells with few mitotic figures and ample osteoid production. Extensive necrosis with partially mineralized osteoid closely associated with neoplastic cells is present.

Amputation and chemotherapy were discussed with the owner, but both were declined. The patient was maintained on deracoxib (Deramaxx, Elanco US Inc., Greenfield, Indiana, United States) $2.2 \mathrm{mg} / \mathrm{kg}$ per os (PO) q 24 hour for 6 weeks until the medication was discontinued due to diarrhoea. The dog was re-evaluated by the primary surgeon 2 weeks later for the gastrointestinal disturbance. At this time, the tibial mass had grown slightly, and the right pelvic limb lameness persisted. After 3 months, the owner requested pain relief medication and the patient was placed on carprofen (Rimadyl, Zoetis LLC, Lincoln, Nebraska, United States) 2.1 $\mathrm{mg} / \mathrm{kg}$ PO q 12 hour for 10 additional weeks.

Eight months following the initial OS diagnosis and 10 weeks after beginning carprofen, the patient was reexamined by the primary care veterinarian. Reportedly, the dog was clinically normal with no right pelvic limb lameness, pain, or tibial swelling. The same board-certified pathologist who viewed the histopathology slides 8 months earlier reviewed the core bone biopsy specimen for a second time and confirmed the initial diagnosis of OS, given the obvious presence of osteoid-producing neoplastic cells (-Fig. 2). The histologic slides were also examined by two additional board-certified pathologists. One of them agreed with the initial OS diagnosis; the second considered chondrosarcoma a possibility but would not rule out OS. Regardless, the three pathologists all agreed with the malignant nature of the bone tumour and reconfirmed the initial bone cancer diagnosis, despite the fact that the lesion had disappeared and the clinical signs had resolved over the period of 8 months.

The patient was re-evaluated by the originally attending surgeon 54 months ( 4.5 years) after the initial OS diagnosis. The dog was not lame or painful and the tibial swelling had completely resolved. A lateral right stifle radiograph ( - Fig. 3) reviewed by a board-certified radiologist revealed no obvious bony lesion. The patient was not sedated for these radiographs and, therefore, only a lateral view was 


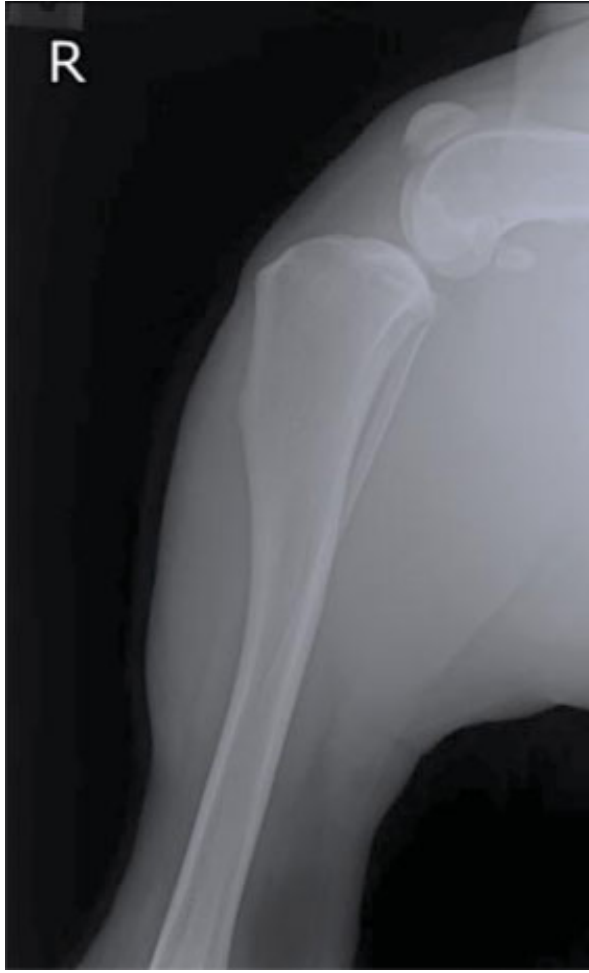

Fig. 3 Lateral stifle radiograph of the patient's right tibia 54 months (4.5 years) after the initial osteosarcoma diagnosis.

performed. After reviewing the radiograph and noting the absence of the previous tibial mass and the lack of any clinical signs, the spontaneous resolution of the OS became evident.

Sixteen months after this re-evaluation and at 102 months ( 8.5 years) of age, the patient was evaluated by the same surgeon because of recent and sudden onset of bilateral pelvic limb lameness. Radiographs of the pelvis revealed only low-degree bilateral hip dysplasia, whereas stifle radiographs showed bilateral severe joint effusion, initial signs of osteoarthritis, and a positive drawer sign characteristic of cruciate ligament disease. These radiographs also exhibited a
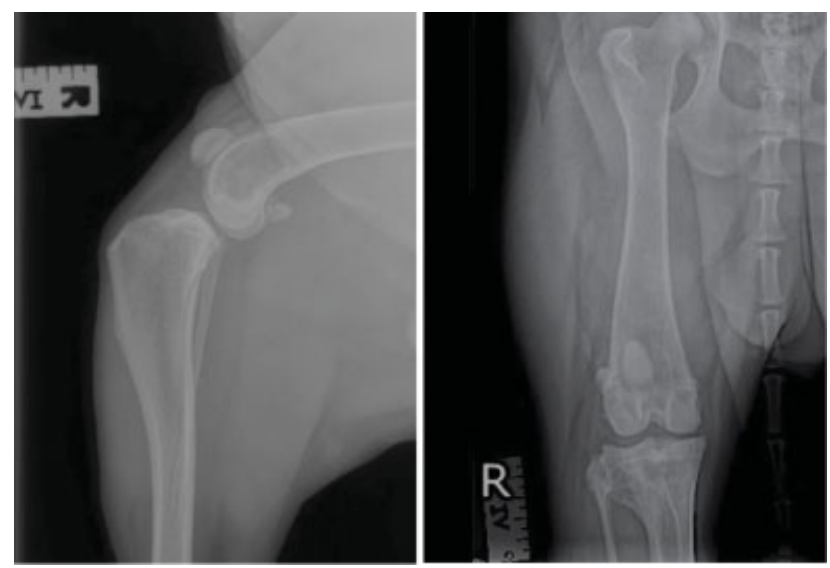

Fig. 4 Orthogonal right stifle radiographs of the patient 70 months (5.8 years) after the initial osteosarcoma diagnosis. The right stifle showed moderate effusion, mild degenerative joint disease and cranial tibial subluxation. The craniocaudal view exhibits a smooth bony protuberance on the lateral aspect of the tibia.
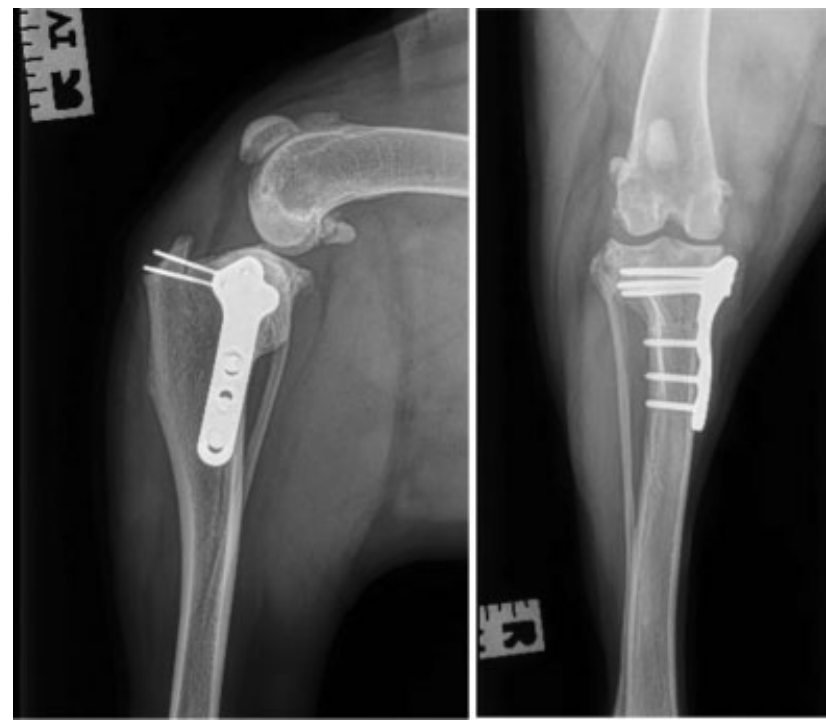

Fig. 5 Orthogonal radiographs of the patient's right stifle 8 weeks after the tibial plateau levelling osteotomy, which revealed normal healing of the tibial osteotomies and unchanged implants.

smooth bony protuberance on the lateral aspect of the proximal right tibia, in the area of the previously regressed bone tumour ( - Fig. 4). A TPLO was performed on the left stifle first, then 2 weeks later, on the right stifle by the same surgeon. The patient had a complete rupture of the cranial cruciate ligaments. During surgery, the proximal tibia appeared normal with no evidence of any pathology. Partial meniscectomies were performed for a medial meniscal injury. A $3.5 \mathrm{~mm}$ TPLO plate $(3.5 \mathrm{~mm}$ Swiss-style stainless steel TPLO plate, Veterinary Orthopedic Implants, St. Augustine, Florida, United States) and six bone screws were used. The tissue planes were closed with Biosyn (Biosyn Monofilament Absorbable Suture, Covidien, Medtronic, Minneapolis, Minnesota, United States) monofilament absorbable sutures and skin staples. The dog was discharged on tramadol (Ultram, Janssen Ortho, LLC, Gurabo Puerto Rico 00778)

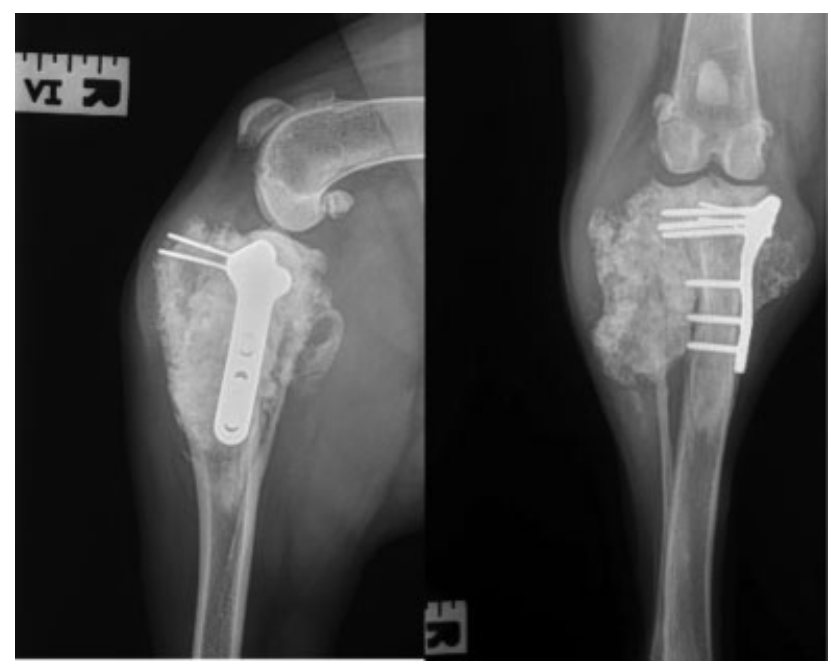

Fig. 6 Orthogonal right stifle radiographs 24 months following the tibial plateau levelling osteotomy, revealing an osteolytic and productive lesion of the right proximal tibia. 


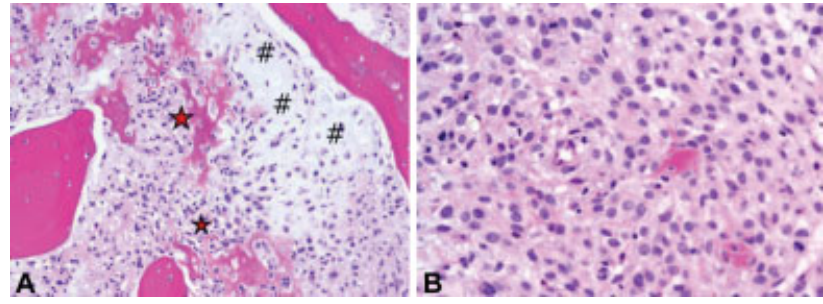

Fig. 7 (A) Histopathology figure (20× magnification) from the right tibial bone biopsy revealing tumour cells with areas of osteoid deposition (stars) as well as some chondroid matrix (\#). (B) Histopathology figure (400× magnification). The neoplastic tissue consists of spindled to polygonal cells with nuclear atypia and high mitotic count consistent with high-grade osteosarcoma. Osteoid production is closely associated with the neoplastic cells.

$1 \mathrm{mg} / \mathrm{kg}$ PO q 12 hour, carprofen $2.1 \mathrm{mg} / \mathrm{kg}$ PO q 12 hour, and cefpodoxime Proxetil (Simplicef, Sandoz, Kundl, Austria) $4.2 \mathrm{mg} / \mathrm{kg}$ PO q 24 hour.

Eight weeks following the second TPLO, follow-up radiographs of both stifles revealed normal healing of the tibial osteotomies with implants in situ (-Fig. 5). The patient was doing well at home with no apparent lameness.

The owner reported that the dog maintained normal activity without lameness following the TPLO surgeries until 24 months later when swelling around the right stifle became apparent at 129 months (10.7 years) of age. The patient was exhibiting mild lameness and there was a firm, large swelling around the proximal right tibia below the stifle-the very same site where OS was identified 8 years earlier. The swelling was mildly painful upon palpation, but the rest of the clinical exam was normal. Radiographs of the right stifle revealed an $8 \times 9 \mathrm{~cm}$ primarily osteoproliferative lesion located around the proximal lateral tibia (-Fig. 6). The lesion was biopsied with an 11-gauge Jamshidi instrument and samples were sent for histopathology examination. One of the board-certified pathologists who had evaluated the previous tumour 8 years ago noted the presence of osteoid and new bone formation associated with neoplastic cells. There was a high mitotic index and typical cellular features consistent with OS (-Fig. 7A and B). The owner declined amputation (again) and treated the dog symptomatically with firocoxib (Previcox, Boehringer Ingelheim, Athens, Georgia, United States) $5 \mathrm{mg} / \mathrm{kg}$ PO q 24 hour and gabapentin (Neurontin, Pfizer, New York City, New York, United States) $5 \mathrm{mg} / \mathrm{kg}$ PO q 12 hour. The tumour grew rapidly and the patient became non-weight bearing with severe swelling of the tibia and oedema of the right pelvic limb. The final evaluation was performed at 132 months ( 11 years) of age, nearly 8.5 years after the original diagnosis. The tumour had grown to over $30 \mathrm{~cm}$ in diameter and the limb was severely swollen. The dog was euthanatized 2 weeks later due to progressive painful swelling, lameness, and inappetence. Post-mortem examination was not performed.

\section{Discussion}

The dog of this report underwent repeated clinical exams, serial radiographs, and multiple biopsies by the same board- certified surgeon, pathologists, and radiologist from 32 to 133 months of age (11 years). We describe herein a radiographically and histopathologically diagnosed appendicular OS which spontaneously regressed over a period of 8 months without therapy, except an analgesic (carprofen). To the authors' knowledge, there are only four other reported cases of OS spontaneously regressing in dogs. Those patients were monitored from 9 months to 2 years after initial diagnosis and later revealed a partial or complete resolution of signs of the tumour disease. The four dogs were diagnosed with primary OS via radiographs and/or biopsy, but lifetime follow-up is not reported. The median expected survival time for appendicular OS is reported to be less than 3 months when analgesics are the only treatments. ${ }^{6,17}$

When spontaneous tumour regression became apparent, an external board-certified veterinary pathologist examined the initial histologic sections again considering that the initial lesion, when the patient was 32 months old, might have been a fracture callus misdiagnosed as OS. Differentiating between an exuberant fracture callus and OS can be difficult when the core biopsies are narrow $(1 \mathrm{~mm})$ as both are spindle cell proliferations with chondroid matrix and osteoid production. However, a callus is unlikely considering the patient had a $6 \mathrm{~cm}$ painful tumefaction at the time of diagnosis that continued to grow in size for at least 5 months, as well as no known history of trauma. Therefore, after careful review of the pathology and clinical data in this case, all consulting specialists supported the initial diagnosis of OS.

While the cause of OS is ill understood, there are risk factors which may induce canine bone cancer. Possible mediators of OS include chemical carcinogens, skeletal disorders, microscopic fractures, metallic implants and ionizing radiation. ${ }^{6}$ OS can also be genetically linked to abnormalities related to tumour protein p53 and retinoblastoma protein. ${ }^{6}$

Large and giant breeds such as Great Danes, Saint Bernards, and Doberman Pinschers are at higher risk for developing appendicular OS compared with small breed dogs. 4,6,12,23 Labrador Retrievers, like this patient, are also at increased risk. ${ }^{23}$ The median age at diagnosis is 7 years, with another smaller peak between 18 and 24 months. ${ }^{3,12,27}$

Spontaneous regression of cancer is rare and the cause is not well understood. One theory includes the immune system's ability to recognize tumour cells as alien or abnormal. ${ }^{28}$ In studying spontaneous regression of breast cancer in humans, CD8 $+\mathrm{T}$ cells were shown activated and may be involved in downregulating malignant cell growth. ${ }^{29}$ The same investigators stated that any needle biopsy could potentially promote such an immune response. It is possible in this reported case that as consequence of the core bone biopsy, a CD8+ response to malignant cells contributed to spontaneous tumour regression, which would be opposed to the general belief that biopsy could enhance tumour spread.

Fracture-induced or metallic implant-associated OS has been recorded in canine patients sporadically throughout the years with the first known case report in 1959. ${ }^{12,23-27}$ One study of 2,464 dogs with metallic osteoimplants showed that 40 dogs had developed OS around implants. ${ }^{30}$ Additional 
reports describe an OS developing 6 years after a TPLO procedure $^{26}$; a Cane Corso is reported to have OS arising 6 years after a tibial tuberosity advancement ${ }^{31}$; and in a Labrador Retriever 12 years after tibial fracture repair. ${ }^{32}$ This latter dog died 1 month following amputation of the tumourcontaining limb. ${ }^{32}$ Fracture-induced or metallic implantassociated OS specifically developing around the TPLO plate has a median occurrence of 2 to 4 years following the procedure. ${ }^{30}$ Factors possibly promoting the development of fracture-related sarcomas include patient sensitivity to the implant, implant corrosion, instability of the fracture or inappropriate bone healing. ${ }^{33}$ It is likely that in our Labrador's case the metal implant was the trigger for the second OS development. However, due to the post-TPLO occurrence in the same location as the first tumour, it is equally possible that the second tumour is not directly linked to the metal implant.

A few case reports have acknowledged the role of nonsteroidal anti-inflammatory drugs (NSAIDs) in relation to spontaneously regressing malignancy. ${ }^{1,13,17,34}$ Carprofen inhibits cyclooxygenases (COX-1 and COX-2) and it may also promote apoptosis of cancer cells. ${ }^{1}$ In a mouse model, it was shown that NSAID-COX-2 inhibitors downregulated osteoclast activity, reduced pain and decreased bone destruction. ${ }^{35}$ In the previous report describing the four dogs with spontaneously regressed OS, three of them were under treatment with NSAIDs at the time of OS diagnosis. ${ }^{1}$ In our case, 5 months after initial OS diagnosis, carprofen was prescribed for 10 weeks; thereafter, all previous clinical signs on the right pelvic limp had resolved. It is possible that the 10-week prescription of carprofen contributed to apoptosis of the cancer cells. Even then, circulating tumour cells can remain dormant and undetected by the immune system until a triggering event such as trauma to the bone (e.g. a TPLO procedure) may result in tumourigenesis and eventually in a fatal tumour disease. ${ }^{36}$

\section{Conclusion}

Spontaneous regression of cancer is a rarely observed and only sporadically recorded phenomenon in both human and veterinary medicine. ${ }^{1,17-21}$ At 32 months of age, the dog of this report was histologically and radiographically diagnosed with tibial OS. Eight months later and after treatment with carprofen for 10 weeks, the tumour had spontaneously and completely regressed. Sixty-three months (5.2 years) later, the dog presented with bilateral cranial cruciate ligament rupture and underwent TPLO procedures. Twenty-four months following surgery, OS occurred in the identical location on the tibia where the tumour had regressed 8 years earlier, confirmed by histopathology and radiographs. The tumour grew rapidly, and the patient was euthanatized 3 months after this second OS diagnosis at 133 months (11 years) of age due to the progression of the tumour disease.

Conflict of Interest

None declared.

\section{Acknowledgment}

The authors would like to thank Dr Jose Ramos-Vara and all the pathologists at the Animal Disease Diagnostic Laboratory, Purdue University College of Veterinary Medicine of West Lafayette, Indiana, United States, for their expertise and assistance with the histopathologic diagnosis.

\section{References}

1 Mehl ML, Withrow SJ, Seguin B, et al. Spontaneous regression of osteosarcoma in four dogs. J Am Vet Med Assoc 2001;219(05): 614-617

2 Withrow SJ, Page RL, Vail DM. Withrow and MacEwen's Small Animal Clinic Oncology. 5th edition St. Louis: Saunders Elsevier; 2007:381-431

3 Ehrhart NP, Ryan SD, Fan TM. Tumours of the skeletal system. In: Withrow S, ed. MacEwen's Small Animal Clinical Oncology. 5th edition St. Louis: Elsevier Saunders; 2012:463-503

4 Brodey RS, Riser WH. Canine osteosarcoma. A clinicopathologic study of 194 cases. Clin Orthop Relat Res 1969;62(62):54-64

5 Mueller F, Fuchs B, Kaser-Hotz B. Comparative biology of human and canine osteosarcoma. Anticancer Res 2007;27(1A):155-164

6 Varshney J, Scott MC, Largaespada DA, Subramanian S. Understanding the osteosarcoma pathobiology: a comparative oncology approach. Vet Sci 2016;3(01):1-15

7 Anfinsen KP, Grotmol T, Bruland OS, Jonasdottir TJ. Breed-specific incidence rates of canine primary bone tumors-a population based survey of dogs in Norway. Can J Vet Res 2011;75(03): 209-215

8 Walter CU, Dernell WS, LaRue SM, et al. Curative-intent radiation therapy as a treatment modality for appendicular and axial osteosarcoma: a preliminary retrospective evaluation of 14 dogs with the disease. Vet Comp Oncol 2005;3(01):1-7

9 Misdorp W. Skeletal osteosarcoma. Animal model: canine osteosarcoma. Am J Pathol 1980;98(01):285-288

10 Tjalma RA. Canine bone sarcoma: estimation of relative risk as a function of body size. J Natl Cancer Inst 1966;36(06):1137-1150

$11 \mathrm{Ru}$ G, Terracini B, Glickman LT. Host related risk factors for canine osteosarcoma. Vet J 1998;156(01):31-39

12 Morello E, Martano M, Buracco P. Biology, diagnosis and treatment of canine appendicular osteosarcoma: similarities and differences with human osteosarcoma. Vet J 2011;189(03):268-277

13 McNeill CJ, Overley B, Shofer FS, et al. Characterization of the biological behaviour of appendicular osteosarcoma in Rottweilers and a comparison with other breeds: a review of 258 dogs. Vet Comp Oncol 2007;5(02):90-98

14 Knecht CD, Priester WA. Musculoskeletal tumors in dogs. J Am Vet Med Assoc 1978;172(01):72-74

15 Heyman SJ, Diefenderfer DL, Goldschmidt MH, Newton CD. Canine axial skeletal osteosarcoma. A retrospective study of 116 cases (1986 to 1989). Vet Surg 1992;21(04):304-310

16 Tohme S, Simmons RL, Tsung A. Surgery for cancer: a trigger for metastases. Cancer Res 2017;77(07):1548-1552

17 Liptak JM, Dernell WS, Ehrhart N, Withrow SJ. Canine appendicular osteosarcoma: diagnosis and palliative treatment. Oncol Comp 2004;26(03):172-183

18 Dissing I, Heerfordt J, Schiødt T. Spontaneous regression of a malignant primary bone tumour. Acta Orthop Scand 1978;49 (01):49-53

19 Carlsen NL. [Spontaneous regression of malignant tumors]. Ugeskr Laeger 1999;161(26):3980

20 Ogihara Y, Takeda K, Yanagawa T, Hirasawa Y. Spontaneous regression of lung metastases from osteosarcoma. Cancer 1994; 74(10):2798-2803 
21 Sabaté JM, Llauger J, Torrubia S, Amores S, Franquet T. Osteosarcoma of the abdominal wall with spontaneous regression of lung metastases. AJR Am J Roentgenol 1998;171(03):691-692

22 Rae MV. Spontaneous regression of a hypernephroma. Am J Cancer 1935;24(04):839-841

23 Withrow SJ, Powers BE, Straw RC, Wilkins RM. Comparative aspects of osteosarcoma. Dog versus man. Clin Orthop Relat Res $1991 ;(270): 159-168$

24 Stevenson S. Fracture-associated sarcomas. Vet Clin North Am Small Anim Pract 1991;21(04):859-872

25 Sinibaldi K, Rosen H, Liu SK, DeAngelis M. Tumors associated with metallic implants in animals. Clin Orthop Relat Res 1976;(118): 257-266

26 Atherton MJ, Arthurs G. Osteosarcoma of the tibia 6 years after tibial plateau leveling osteotomy. J Am Anim Hosp Assoc 2012;48 (03):188-193

27 Brodey RS. The use of naturally occurring cancer in domestic animals for research into human cancer: general considerations and a review of canine skeletal osteosarcoma. Yale J Biol Med 1979;52(04):345-361

28 Jessy T. Immunity over inability: the spontaneous regression of cancer. J Nat Sci Biol Med 2011;2(01):43-49

29 Takayama S, Satomi K, Yoshida M, et al. Spontaneous regression of occult breast cancer with axillary lymph node metastasis: a case report. Int J Surg Case Rep 2019;63:75-79
30 Sartor AJ, Ryan SD, Sellmeyer T, Withrow SJ, Selmic LE. Biinstitutional retrospective cohort study evaluating the incidence of osteosarcoma following tibial plateau levelling osteotomy (2000-2009). Vet Comp Orthop Traumatol 2014;27(05):339-345

31 Sharma S, Boston SE, Riddle D, Isakow K. Osteosarcoma of the proximal tibia in a dog 6 years after tibial tuberosity advancement. Can Vet J 2020;61(09):946-950

32 Isaka M, Kokubo D, Sakai T. The occurrence of osteosarcoma after tibial fracture repair in a dog. Open Vet J 2021;11(01):11-13

33 Gilley RS, Hiebert E, Clapp K, et al. Long-term formation of aggressive bony lesions in dogs with mid-diaphyseal fractures stabilised with metallic plates: incidence in a tertiary referral hospital population. Front Vet Sci 2017;4(03):3

34 Mullins MN, Lana SE, Dernell WS, Ogilvie GK, Withrow SJ, Ehrhart EJ. Cyclooxygenase-2 expression in canine appendicular osteosarcomas. J Vet Intern Med 2004;18(06):859-865

35 Sabino MA, Ghilardi JR, Jongen JLM, et al. Simultaneous reduction in cancer pain, bone destruction, and tumor growth by selective inhibition of cyclooxygenase-2. Cancer Res 2002;62(24): 7343-7349

36 Rossi E, Fabbri F. CTCs 2020: great expectations or unreasonable dreams. Cells 2019;8(09):989 